\title{
Mediated presence: virtual reality, mixed environments and social networks
}

\author{
Anna Spagnolli · Matthew Lombard • \\ Luciano Gamberini
}

Received: 30 June 2009/Accepted: 1 July 2009/Published online: 25 July 2009

(C) Springer-Verlag London Limited 2009

The processes through which human beings establish and experience presence in their life environment, especially when this environment is increasingly mediated and even generated by technologies, is a fascinating subject of scholarly investigation. Our expanding abilities to bring presence from the "real" world and from "real" human interactions to mediated worlds and mediated social relations represents an important chance to enrich human experience. Designing technologies and imagining practices to modify, prolong and reconfigure the possibilities of being present has been a continuous endeavour of the human species, from early attempts at constructing communication and transportation devices to the many current technologies we continue to develop to reach other places and people. Virtual business meetings via telepresence conferencing systems, remote medical consultations and even surgery, the recording and transmission of political unrest to the global community, web and virtual worldbased communities based on common concerns and interests, and compelling interactive single and massively multiplayer electronic games only begin to illustrate the power of technology-mediated presence, or telepresence. Exploring empirically and reflecting theoretically upon the conditions through which presence (and absence) are established, playing with presence possibilities, augmenting them, identifying the events and resources that affect them in different ways all help us better understand and

\footnotetext{
A. Spagnolli · L. Gamberini

University of Padova, Padua, Italy

M. Lombard ( $\square)$

School of Communications and Theater,

Temple University, Philadelphia, USA

e-mail: lombard@temple.edu
}

reach our potential to communicate effectively and better understand the human experience.

This special issue illustrates the variety of research questions and approaches being used to explore mediated presence in virtual reality, mixed environments and social networks. The opportunity to create the issue emerged during PRESENCE 2008, the 11th International Workshop on Presence. An open call to conference attendees and others yielded more than 30 submissions. After several revisions, and thanks to more than 90 experts who kindly served as reviewers, we are able to offer a remarkable collection of papers in this and a future issue, that provide an overview of the state of the art in the field. They include both theoretical essays and empirical studies and present new advances in well established lines of investigation as well as new theoretical and methodological approaches.

Three papers all have in common the analysis of participants' action as a method to investigate presence and social presence.

The first in this group is "Afforded actions as a behavioral assessment of physical presence in virtual environments" by Jean-Claude Lepecq, Lionel Bringoux, Jean-Marie Pergandi, Thelma Coyle, and Daniel Mestre. The rationale behind the paper is that users who feel present in a virtual environment will behave as if they were in the environment. In the setting devised by the authors, the virtual movement produced by an avatar in entering a virtual door was accompanied by a rotation of the users' real shoulders and this varied according to the actual size of the users' shoulders and the size of the virtual door. While the study was only conducted with male participants and with a virtual environment uniquely designed to study the movement under investigation, its systematic results suggest that the sensorimotor affordances of a virtual environment can be used to objectively assess the achievement 
of presence. (It is worth noticing that a relationship between sense of presence and the production of a physical behavior, i.e. looking at a virtual window, is also hinted to by Wallach and colleagues in another paper of this special issue, although not the central focus of their work.)

Moving the focus to social presence, the next paper proposes a method to augment presence in groups of users and to measure this augmentation by deploying action as the datum. In "Presence in the age of social networks: Augmenting mediated environments with feedback on group activity," Francesco Martino, Roberto Baù, Anna Spagnolli and Luciano Gamberini motivate the interest in social presence at a group level with the current diffusion of mediated environments in which social interaction is not only one-to-one, but also at the larger network level. They address social presence not in terms of feelings, but of actions, as behavioral engagement ("being socially present") in order to capture its actual achievement at a pragmatic level. In the study they describe, participants are behaviorally engaged with the group when they exchange information about their task, a treasure hunt; their communication activity is recorded and quantified, both to provide the participants feedback and as the basis for measuring their level of behavioral engagement. The results support the possibility of increasing behavioral engagement with feedback based on Social Network Analysis, and confirm the opportunity to tune the measurement technique to the actual dimension of social presence one is measuring: in this case, action-based techniques (automatically logged communication events) proved more helpful than selfreported ones to capture behavioral engagement.

In "Social cooperation and competition in the mixed reality space eXperience Induction Machine XIM," Martin Inderbitzin, Sytse Wierenga, Aleksander Valjamae, Ulysses Bernardet, and Paul FMJ Verschure report on their modeling of group behavior by automatically measuring one dimension of the interaction, interpersonal distance. The authors' interest is not so much in the measure of social presence as such, but in identifying patterns of physical presence characterizing specific activity outcomes, and in applying systematic measurements to do so. As in the two articles discussed above, the task and mediated environment are consonant with the specific measurement procedure adopted. The mixed reality environment used is an instrumented room whose floor can sense the presence of the players (and react to it), and the activity is a football (soccer) game with two teams. The analysis of gameplay in the environment identified specific patterns of intra-team member distance during activities leading to a goal. The study suggests a promising approach for the study of complex social phenomena that involve physical interactions in a variety of mixed reality environments.
Two papers in this special issue provide an opportunity to reflect on the nature of presence and on the methods usually adopted to study it. While presence is often informally defined as 'the sense of being there' in a mediated environment and an official, shared definition has been established by the International Society for Presence Research, discussion of the nature of presence has always characterized the research field, in an attempt to capture all of the facets that characterize the phenomenon and take advantage of different theoretical orientations of the scholars investigating them. Some of these definitions, including the one adopted by ISPR, have been followed by research programs substantiating them empirically, thereby increasing the heuristic value of the proposed definition.

In his article here, "Is Presence a Technology Issue? Some insights from Cognitive Sciences," Giuseppe Riva uses insights from psychology and neuro-psychology to advance a conceptual framework for presence that addresses several recent questions about the nature of presence phenomena. His definition of "a core neuropsychological phenomenon whose goal is to produce a sense of agency and control" moves from a focus on action and individual perceptions to a focus on intentions and interactions among individuals, while emphasizing the continuity of experiences both mediated and not mediated by technology, and more fully considering the role of individual differences and contexts in generating presence experiences. The model suggests testable predictions and design choices for evoking satisfying and productive telepresence experiences.

Taking a similarly broad view, this time of telepresence research, in "Triangulation in Practice" Phil Turner and Susan Turner advocate the use of an approach to advancing our knowledge that is often used in fields where study is difficult, demanding or contentious. Triangulation is the procedure through which different definitions and theories, methods, data, and investigators are used in conjunction in order to achieve a more comprehensive view of a phenomenon and to reciprocally validate each other. The authors encourage a more frequent recourse to this solution, and strengthen their argument by providing the example of a study they carried out. As exemplified also by the work of Martino and his colleagues in this issue, where action-based and self-reported measures are deployed to investigate a type of social presence, it is necessary to properly tune the method to the phenomenon under investigation in order to develop a valid, discriminative measurement technique. The authors recommend avoiding triangulation as a mere juxtaposition of methods and definitions, and invite us instead to pay careful attention to the epistemological premises of the different techniques triangulated. 
An uncommon application of social presence and classic teleoperation is described in "Co-presence, collaboration, and control in environmental studies: A Second Life based approach" by Songpol Attasiriluk, Arturo Nakasone, Wisut Hantanong, Rui Prada, Pizzanu Kanongchaiyos and Helmut Prendinger. The authors developed an interactive, real-time, avatar-based system using the popular Second Life platform that supports collaborative decision making and learning in a virtual environment that corresponds to a real one. By connecting the virtual environment to sensors and actuators in actual rice paddies and vineyards, experts and laymen are able to "meet at" and discuss, affect, and learn from the real environment. The technical characteristics of the system are illustrated, accompanied by a short experiment in which users experienced co-presence. The approach holds great potential to create seamless communication between the real and virtual worlds in a variety of contexts.

Finally, in "Attachment and sense of presence in a virtual environment," Helene Wallach, Marilyn Safir, and Idan Almog explore individual differences in attachment styles to predict which people are more likely to experience presence, and thereby, it is hoped, successful treatment, in a therapeutic virtual environment (a virtual airplane flight). An experiment yielded results that provide valuable links between these new individual difference variables and presence, with the potential to increase the effectiveness of an important practical application of mediated presence.

A second set of articles selected in response to our call will be published in a future issue of Virtual Reality. We would like to thank the journal for providing this opportunity and for following the project with care and attention. We are extremely grateful to the many reviewers who have contributed their valuable time to read (and re-read) the manuscripts, and to Co-Editor-in-Chief Dan Ballin for his support. We hope that readers will find value in the work included here and continue to follow advances in the field of presence both in subsequent issues of this journal and elsewhere (a gateway to much more information about presence and telepresence is the web site of the International Society for Presence Research, http://ispr.info/). 